\title{
UNIVERSITYOF
}

FORWARD

THINKING

WESTMINSTER用

WestminsterResearch

http://www.westminster.ac.uk/westminsterresearch

\section{Electricity Price Forecasting using Asymmetric Fuzzy Neural} Network Systems

\author{
Alshejari, A. and Kodogiannis, Vassilis
}

This is a copy of the author's accepted version of a paper subsequently to be published in the proceedings of the 2017 International Conference on Fuzzy Systems (FUZZ-IEEE 2017). Naples, Italy 09 to 12 Jul 2017, IEEE.

It is available online at:

https://dx.doi.org/10.1109\%2FFUZZ-IEEE.2017.8015436

(C) 2017 IEEE . Personal use of this material is permitted. Permission from IEEE must be obtained for all other uses, in any current or future media, including reprinting/republishing this material for advertising or promotional purposes, creating new collective works, for resale or redistribution to servers or lists, or reuse of any copyrighted component of this work in other works.

The WestminsterResearch online digital archive at the University of Westminster aims to make the research output of the University available to a wider audience. Copyright and Moral Rights remain with the authors and/or copyright owners.

Whilst further distribution of specific materials from within this archive is forbidden, you may freely distribute the URL of WestminsterResearch: ((http://westminsterresearch.wmin.ac.uk/)).

In case of abuse or copyright appearing without permission e-mail repository@westminster.ac.uk 


\title{
Electricity Price Forecasting using Asymmetric Fuzzy Neural Network Systems
}

\author{
Abeer Alshejari, Vassilis S. Kodogiannis \\ Faculty of Science and Technology \\ University of Westminster \\ London, United Kingdom \\ V.Kodogiannis@,westminster.ac.uk
}

\begin{abstract}
Electricity price forecasting is considered as an important tool for energy-related utilities and power generation industries. The deregulation of power market, as well as the competitive financial environment, which have introduced new market players in this field, makes the electricity price forecasting problem a demanding mission. The main focus of this paper is to investigate the performance of asymmetric neuro-fuzzy network models for day-ahead electricity price forecasting. The proposed model has been developed from existing Takagi-Sugeno-Kang fuzzy systems by substituting the IF part of fuzzy rules with an asymmetric Gaussian function. In addition, a clustering method is utilised as a pre-processing scheme to identify the initial set and adequate number of clusters and eventually the number of rules in the proposed model. The results corresponding to the minimum and maximum electricity price have indicated that the proposed forecasting scheme could be considered as an improved tool for the forecasting accuracy.
\end{abstract}

Keywords-Electricity price forecasting; neurofuzzy systems; neural networks; clustering; prediction

\section{INTRODUCTION}

During the past two decades, liberalization and deregulation policies have been applied in energy sector in most of EU countries. With the introduction of abolishing the public nature of electric power industry, the price of electricity has become probably the central point of all activities in this market [1]. Electricity price forecasting is a challenging task and is considered as a very important parameter in such competitive electricity market.

However the problem of electricity price forecasting is, in some ways, different from that of load forecasting. Although both load and the price are linked, such relation is mainly non-linear. Electricity load is affected by parameters such as seasonal changes in energy demand, energy-saving behavior of energy consumers and mainly by the fact the electricity load is not a substitute for storability. Price, alternatively, is influenced by the same factors as well as additional features such as financial regulations, competitors' load pricing, dynamic market factors, and other macro/micro economic conditions. Hence, electricity price can be considered as more volatile than the electricity load. It is worth mentioned, that during the introduction of dynamic pricing strategies, electricity prices become even more volatile, where the daily average price were changed by up to
$50 \%$ while other commodities exhibited about 5\% change [2].

Both power market players as well as independent operator regulators (ISO) are alarmed with such price evolution. Market electricity price prediction is thus important information for producers' production planning and price bidding strategies. Obviously, various methods have been adopted for the forecasting of future prices. One approach for market behaviour prediction is the usage of regression methods. The fundamental idea is to utilise historical electricity prices, power load forecast as well as temperature information to predict the market-clearing price (MCPs). However, the utilisation of such simple linear regression model cannot capture the complicated nonlinear dynamic relation between load and electricity prices [3]. Classic ARMA models on the other hand utilise historical time-series data, but again they fail to consider the effect of other factors on electricity prices. With the presence of small number outliers, the fitting error of such model may greatly increase. Hence, this "defect" limits its application extensively.

Neural Network (NNs) and other intelligent schemes have enjoyed a great applicability in electricity price forecasting, which is due to their simple and flexible architecture. Among existing intelligent schemes, generalized regression neural network (GRNN) incorporated with principal components analysis (PCA) have shown potential in electricity price forecasting [4]. Although, according to literature, the majority of applied case studies are referred to day-ahead predictions, the MLP network has been utilized in hour-ahead time forecasting [5]. The role of MLP is to enhance the performance of classic time series models (for example an ARIMA). RBF is another type of NNs that is utilized in the case study of [6]. This specific model is able to simulate complex nonlinear relationships, sometimes with greater accuracy than MLP networks. Support Vector Machines (SVMs) provide also a non-linear mapping of the dataset into a high-dimensional space. The boundaries of this new high-dimensional space are distinguished by linear functions. SVMs provide a global solution to a problem unlike MLPs which operate by minimising problem's objective function. Such interesting characteristic has been acknowledged in many case studies related to the electricity load and price forecasting area [7]. 
In one of the first applications utilising fuzzy logic to electricity price forecasting, a combination of fuzzy c-means clustering and a neural recurrent network has been considered [8]. Another approach in electricity price forecasting is the use of hybrid neurofuzzy systems. An adaptive-network-based fuzzy inference system (ANFIS) has been investigated and results proved that such scheme is superior to MLP approaches [9].

In the majority of electricity price forecasting studies, especially for the hourly price case, only one model is normally utilized to forecast the next 24 hourly prices. However, it is a rather difficult task to associate all the characteristics of 24 different hourly prices by a single model. Thus, the model may become under-fitting for some hourly predictions, while at the same time, it may become over-fitting for some others, which eventually leads to unsatisfactory results. An obvious disadvantage of such approach is related to the high complexity of the network structure (i.e. a system with 24 output nodes) in terms of training time and performance. Alternatively, a recurrent structure could provide similar characteristics, however in practice its performance would be deteriorated due to the feedback error accumulation. An alternative approach has been proposed in recent past [10] and it has been adopted also in this paper. The core of the proposed modular forecasting system is the 24 multi-input-single-output (MISO) modeling blocks. One of the advantages of the proposed modular system is its possible use also for longrange forecasting schemes.

In this paper, neurofuzzy models are considered to compute the forecasted price in ISO New England market. The ISO New England market is coordinated by an independent system operator (ISO) (http://www.iso-ne.com). It has been observed that although the daily load curves retain a similar pattern, the equivalent daily price curves are however volatile. Hence, the forecasting of Locational Marginal Prices (LMPs) becomes important as it helps the determination of the bidding market strategies as well as in risk management. In this research, the training/testing data set was created from the period 2006-2007. Both training and testing sets were classified into 24 time series, each one corresponding to a different hour of the day. More specifically, 600 data were allocated to training subset, while 123 data for the testing one.

An Asymmetric Gaussian Fuzzy Inference Neural Network (AGFINN), utilizing a Takagi-Sugeno-Kang (TSK) structure, has been considered as an identification model for electricity price forecasting. Unlike the ANFIS system, AGFINN involves a clustering component which reduces the number of fuzzy rules, minimizing thus the "curse of dimensionality" problem. A fuzzy c-means (FCM) clustering algorithm has been applied at the sample data in order to categorize feature vectors into clusters. In the following result section, only results that correspond to hours with the maximum $(22: 00 \mathrm{~h})$ and minimum $(04: 00 \mathrm{~h})$ electricity prices are illustrated. The proposed modeling scheme is compared against ANFIS, AFLS and MLP NN forecasting schemes utilized for the same case study in order to evaluate its performance as an efficient prediction scheme.

\section{ASYMMETRIC NEUROFUZZY MODEL (AGFINN)}

In this section, the proposed Asymmetric Gaussian Fuzzy Inference Neural Network (AGFINN) is presented an alternative neurofuzzy modelling approach. Although AGFINN follows the classic TSK-defuzzification structure, it includes a FCM clustering scheme for structural / initialization purposes. Although standard symmetric Gaussian membership functions has been utilised widely, AGFINN utilizes an asymmetric function acting as input linguistic node. Since the asymmetric Gaussian membership function's variability and flexibility are higher than the standard one, it can partition input space more effectively [11]. In this paper, AGFINN has been optimised through the gradient descent learning algorithm. The architecture of the proposed neurofuzzy (NF) network shown in Fig 1 consists of five layers.

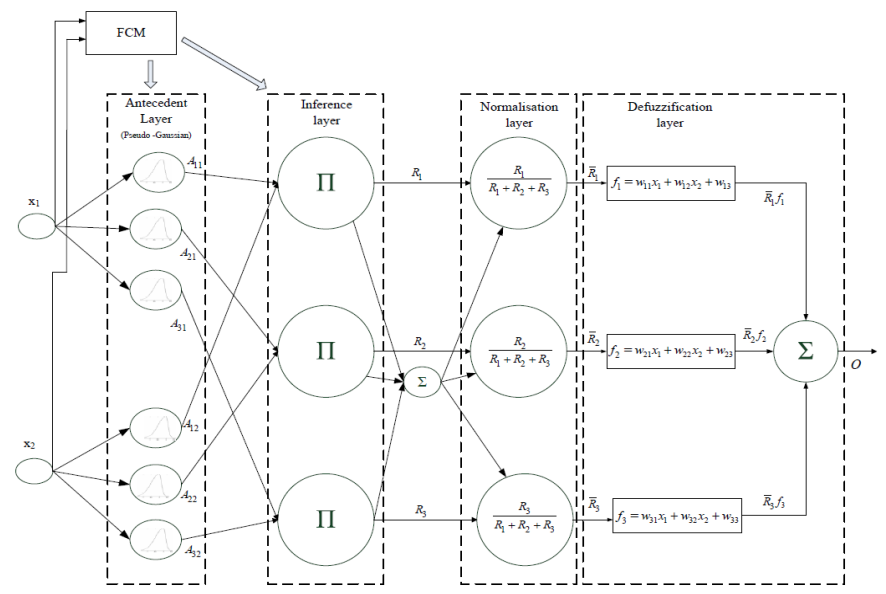

Fig. 1. Structure of AGFINN system

The first three layers $L_{1}, L_{2}$ and $L_{3}$ correspond to IF part of fuzzy rules whereas layer $\mathrm{L}_{5}$ contains information about THEN part of these rules and perform the defuzzification task. In layer $\mathrm{L}_{4}$ a normalization process is performed for all rules derived from $\mathrm{L}_{3}$.

\section{A. FCM Clustering Algorithm}

Fuzzy c-means (FCM) clustering is probably the most wellknown fuzzy unsupervised clustering algorithm which is based on minimizing an objective function that represents the distance from any given data point to a cluster centre weighted by that data point's membership value. Given $n$ data patterns, $x_{1}, x_{2}, \ldots, x_{n}$, fuzzy clustering partitions the data patterns into $c$ clusters which centred at $c_{i}$. The objective function for FCM is defined by

$$
\sum_{\mathrm{i}=1}^{\mathrm{c}} \sum_{\mathrm{j}=1}^{\mathrm{n}} \mu_{\mathrm{ij}}^{\mathrm{m}} \mathrm{d}_{\mathrm{ij}}^{2}, \quad 1 \leq \mathrm{i} \leq \mathrm{c}
$$

where $\mu_{\mathrm{ij}}$ is the degree of membership of object $\mathrm{j}$ in cluster $\mathrm{i}, \mathrm{m}$ is the fuzzy weighting exponent varying in the range $[1, \infty]$ and $\mathrm{d}_{\mathrm{ij}}$ denotes the Euclidean distance between $\mathrm{x}_{\mathrm{j}}$ and $c_{i}$. The membership $\mu_{i j}$ and the cluster centres $c_{i}$ are calculated by the following equations: 


$$
\begin{gathered}
\mu_{\mathrm{ij}}=\left[\sum_{\mathrm{k}=1}^{\mathrm{c}}\left(\frac{\mathrm{d}_{\mathrm{ij}}}{\mathrm{d}_{\mathrm{kj}}}\right)^{(2 /(\mathrm{m}-1))}\right]^{-1}, 1 \leq \mathrm{i} \leq \mathrm{c}, \quad 1 \leq \mathrm{j} \leq \mathrm{n} \\
\mathrm{c}_{\mathrm{i}}=\frac{\sum_{\mathrm{j}=1}^{\mathrm{n}} \mu_{\mathrm{ij}}^{\mathrm{m}} \mathrm{x}_{\mathrm{j}}}{\sum_{\mathrm{j}=1}^{\mathrm{n}} \mu_{\mathrm{ij}}^{\mathrm{m}}}
\end{gathered}
$$

In $\mathrm{FCM}, \mathrm{c}_{\mathrm{i}}$ is updated via an interactive procedure, using last iteration's membership values. This algorithm shifts objects between clusters until the objective function cannot be decreased further. In the present study, cluster centres have been utilized as initial values for the centres of fuzzy membership functions, while the number of if-then rules for AGFINN modelling is equal to the number of clusters obtained through FCM approach. The spread values for each membership function $\sigma_{i j}$ are initialized according to

$$
\sigma_{i j}=\left(\sum_{k=1}^{n} u_{i k}\left(x_{k j}-c_{i j}\right)^{2} / \sum_{k=1}^{n} u_{i k}\right)^{1 / 2}
$$

These values are calculated based on the matrix $\mathbf{U}$, where its elements correspond to the fuzzy memberships of input $\boldsymbol{x}_{\boldsymbol{k}}$ in the $i^{\text {th }}$ cluster and have centre values obtained again from FCM.

\section{B. Feed-forward analysis of AGFINN}

The clustering algorithm provides the fuzzy c-partition of the sample data and practically generates the fuzzy rules base for the AGFINN scheme. Fuzzy IF-THEN rules can be written in the following form:

$$
\operatorname{IF}\left(x_{1} \text { is } U_{1}^{i} \text { AND....AND } x_{q} \text { is } U_{q}^{i}\right) \operatorname{THEN}\left(y=w_{0}^{i}+w_{1}^{i} x_{1}+. .+w_{q}^{i} x_{q}\right)
$$

where $U$ are fuzzy sets defined based on c-partition of learning data $\mathrm{X}$. The structure of the AGFINN is explained below layer by layer:

- Layer 1: This layer is the input layer. Nodes at this layer simply forward the input signals $x_{1}, x_{2}, \ldots, x_{n}$ to $\mathrm{L}_{2}$.

- Layer 2: This layer is the fuzzification layer, and its nodes are associated with the fuzzy sets used in the antecedent parts of the fuzzy rules. Each fuzzification node determines the degree to which an input belongs to the node's fuzzy set. The outputs of this layer are the values of the asymmetric Gaussian membership function (MF) for the input values.

$$
\begin{aligned}
& A_{i j}=\exp \left(-\left(\frac{x_{i}-c_{i j}}{\sigma_{i j}^{\text {left }}}\right)^{2}\right) U\left(x_{i} ;-\infty, c_{i j}\right)+ \\
& \exp \left(-\left(\frac{x_{i}-c_{i j}}{\sigma_{i j}^{\text {right }}}\right)^{2}\right) U\left(x_{i} ; c_{i j}, \infty\right) \\
& \text { where } U\left(x_{i} ; a, b\right)= \begin{cases}1 & \text { if } a \leq x_{i}<b \\
0 & \text { otherwise }\end{cases}
\end{aligned}
$$

From the above equation, it is obvious that the proposed MF utilizes two spreads, namely $\sigma_{i j}^{\text {left }}$ and $\sigma_{\mathrm{ij}}^{\text {right }}$ respectively. Both of these parameters transform the traditional Gaussian function to a more asymmetric style which can provide greater flexibility from the original one. A schematic of the proposed MF is shown in Fig. 2.

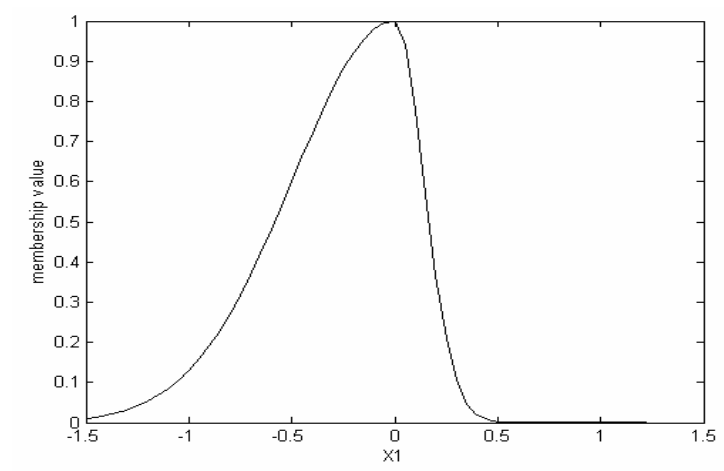

Fig. 2. Asymmetric membership function

- Layer 3: This layer is the firing strength calculation layer. Each fuzzy rule's antecedent part has AND connection operator, thus firing strength is calculated through the product T-norm operator. In this case, the multiplication has been used, and the output of this layer has the following form:

$$
R_{j}=\prod_{i}^{n} A_{j i}\left(x_{i}\right)
$$

- Layer 4: This layer is the normalization layer. Each node in this layer calculates the normalized activation firing of each rule by:

$$
\bar{R}_{i}=\frac{R_{i}}{\sum_{j=1}^{c} R_{j}}
$$

- Layer 5: This layer is associated with the defuzzification part of the AGFINN. Each node at this layer combines the output of each node in $\mathrm{L}_{4}$ by algebraic sum operation after being multiplied by the output weight value $f_{j}$ :

$$
O=\sum_{j=1}^{c} f_{j} \bar{R}_{j}
$$

where $\mathrm{f}_{\mathrm{j}}=\mathrm{w}_{\mathrm{j} 1} \mathrm{x}_{1}+\ldots+\mathrm{w}_{\mathrm{jn}} \mathrm{x}_{\mathrm{n}}+\mathrm{w}_{\mathrm{j}(\mathrm{n}+1)}$ represent the "consequent parameters" of the TSK-style defuzzification scheme.

The learning algorithm of AGFINN utilises gradient descent (GD) method for optimization the various network parameters. During the backward "training" passes, the error signals are calculating from the output layer backward to the premise (i.e. membership) layers, and parameters at both defuzzification and fuzzification sections are fine-tuned. 


\section{RESULTS \& DISCUSSION}

Electricity price is a nonlinear problem with many input variables, including past own values as well as past and forecasted values of any exogenous variables such as electricity consumption. To deal with this fact, three different models have been considered for this study, in order to extract conclusions about the most appropriate forecasting scheme. In general, historical values of the parameter under study have been considered as input candidates for forecasting problems. In electricity price analysis, load factor has been considered as the most important external variable. In this study, we assume that next day's forecasted load is also available. There is a similarity between price and load parameters. While the load level rises, a constant increase of price is observed too.

\section{A. Model A}

The objective of this first model is to examine the simple configuration, used by various researchers, where electricity prices at previous days and hours, as well as forecasted (for the targeted hour/day) load demand are utilized as input variables. Thus, for electricity price modelling for a specific hour (i) and day (j), the following five input variables have been considered:

\section{Target:}

Price $(i, j)$ : electricity price at the $i^{\text {th }}$ hour on the $(j)^{\text {th }} d a y$,

\section{Inputs:}

Price $(i, j-1)$ : price at the $i^{\text {th }}$ hour on the $(j-1)^{\text {th }}$ day,

Price $(i, j-2)$ : price at the $i^{\text {th }}$ hour on the $(j-2)^{\text {th }}$ day,

Price $(i-1, j-1)$ : price at the $(i-1)^{\text {th }}$ hour on the $(j-1)^{\text {th }}$ day,

Price $(i-2, j-1)$ : price at the $(i-2)^{\text {th }}$ hour on the $(j-1)^{\text {th }}$ day,

Load $(i, j)$ : electricity load at the $i^{\text {th }}$ hour on the $j^{\text {th }}$ day,

Based on this configuration, AGFINN model has been involved in forecasting the maximum $(22 \mathrm{~h})$ and minimum (04h) price respectively. Best results were produced by including 20 fuzzy rules for the case of $22 \mathrm{~h}$, while 15 rules was considered as adequate number for the case of $04 \mathrm{~h}$. Although the classic GD method utilized as a learning scheme, the training time was completed in less than 1000 epochs, much faster from the equivalent time used to train the MLP NN. The performance of the forecasting model was determined by the root mean squared error (RMSE), the Mean absolute percentage error (MAPE) (\%) and finally and the standard error of prediction (SEP).

TABLE I

PERFORMANCE INDICES

\begin{tabular}{|l|c|c|}
\hline \multirow{2}{*}{$\begin{array}{c}\text { Statistical index for AGFINN } \\
\text { (Model A) }\end{array}$} & \multicolumn{2}{|c|}{ Testing Data sets } \\
\cline { 2 - 3 } & $\mathbf{2 2 h}$ & $\mathbf{0 4 h}$ \\
\hline Root mean square error (RMSE) & 7.9028 & 3.3783 \\
\hline Mean absolute percentage error (MAPE) (\%) & 5.0385 & 7.0090 \\
\hline Standard error of prediction (SEP) (\%) & 6.5310 & 7.5578 \\
\hline
\end{tabular}

The complete results for the hours with minimum and maximum electricity price, are illustrated in Table I. The
RMSE index is calculated between the desired and output values and then averaged across all data and it can be used as an estimation of the goodness of fit of the models. It can also provide information about how consistent the model would be in the long run. The MAPE term is the average absolute percent error for each time period or forecast minus actual, divided by actual. The SEP index is determined as the relative deviation of the mean prediction values and it has the advantage of being independent on the magnitude of the measurements. Based on these indices, the AGFINN scheme achieved a very good performance, especially for the case of maximum price [12].

In order to evaluate the goodness of the current performance of the proposed AGFINN scheme, a comparison against $\mathrm{NN}$ and neurofuzzy models that have been employed for the specific datasets has been carried out. Table II provide a summary of those statistical performances. More specifically, the AGFINN scheme has been compared against a multilayer perceptron (MLP) and neurofuzzy (NF) ANFIS and AFLS systems.

TABLE II

PERFORMANCE INDICES - COMPARISON

\begin{tabular}{|l|c|c|c|}
\hline Statistical index (22h) & AFLS & ANFIS & MLP \\
\hline Root mean square error (RMSE) & 8.6060 & 10.3569 & 12.8880 \\
\hline Mean absolute percentage error (MAPE) (\%) & 5.4587 & 6.3012 & 7.4165 \\
\hline Standard error of prediction (SEP) (\%) & 7.1122 & 8.5591 & 10.6508 \\
\hline \multicolumn{3}{|l|}{} \\
\hline Statistical index (04h) & AFLS & ANFIS & MLP \\
\hline Root mean square error (RMSE) & 4.5427 & 7.5882 & 10.4905 \\
\hline Mean absolute percentage error (MAPE) (\%) & 9.4320 & 13.6217 & 21.3592 \\
\hline Standard error of prediction (SEP) (\%) & 10.1630 & 16.9763 & 23.4693 \\
\hline
\end{tabular}

The "Adaptive Fuzzy Logic System" (AFLS) model is a advanced MIMO NF systems which incorporates a novel defuzzification scheme, while differs from conventional fuzzy rule-table approaches that utilize the "look-up table" concept [13]. The AFLS scheme does not follow classic TSK's architecture, as the number of memberships for each input variable is directly associated to the number of rules, hence, the "curse of dimensionality" problem is significantly reduced. The fuzzification section in AFLS is similar to AGFINN, with the exception of the FCM clustering step as well as the absence of asymmetric MFs. Similar to AGFINN and MLP, AFLS also utilizes the same GD learning method for training. For this specific case study, 20 fuzzy rules for the case of $22 \mathrm{~h}$, and 15 rules for the case of $04 \mathrm{~h}$ were used as a final configuration. Results shown at Table II reveal that AFLS could be considered as the closest to AGFINN in terms of performance. An MLP network was also constructed for this case study, using the same input vector. After a few trials, utilizing different internal structures, a NN was implemented with two hidden layers (with 20 and 8 nodes respectively). Although AGFINN, AFLS and MLP share the same learning training algorithm, the different "philosophy" in building the neurofuzzy architecture, allowed those systems to achieve a superior performance. Finally an ANFIS NF model has been constructed, utilising 
32 fuzzy rules. As the number of MFs in AGFINN is equal to the numbers of rules, this architecture has advantages over the classic ANFIS model. The increased number of Gaussian membership functions increases the localization of the input signal while in the same time maintains the required number of rules at low level.

\section{B. Model B}

Research has indicated that current hour electricity price shows a high correlation with those of hour h-24 and h-168, a fact that indicates some daily and weekly periodicity. The objective of this model is to investigate this specific issue. No exogenous input variables are considered in this specific case study. Thus, for electricity price modeling for a specific hour (i) and day (j), the following six input variables have been considered:

\section{Target:}

Price $(i, j)$ : electricity price at the $i^{\text {th }}$ hour on the $(j)^{\text {th }}$ day,

\section{Inputs:}

Price $(i, j-1)$ : price at the $i^{\text {th }}$ hour on the $(j-1)^{\text {th }}$ day,

Price $(i, j-2)$ : price at the $i^{\text {th }}$ hour on the $(j-2)^{\text {th }}$ day,

Price $(i, j-3)$ : price at the $i^{\text {th }}$ hour on the $(j-3)^{\text {th }}$ day,

Price $(i, j-7)$ : price at the $i^{\text {th }}$ hour on the $(j-7)^{\text {th }}$ day,

Price $(i-1, j-1)$ : price at the $(i-1)^{\text {th }}$ hour on the $(j-1)^{\text {th }}$ day,

Price $(i-2, j-1)$ : price at the $(i-2)^{\text {th }}$ hour on the $(j-1)^{\text {th }}$ day,

The complete results for the hours with minimum and maximum electricity price, for the AGFINN case are illustrated in Table III. The information related to weekly periodicity indeed resulted in an improved forecasting performance compared to Model A. Best results were produced by including 25 fuzzy rules for the case of $22 \mathrm{~h}$, while 20 rules were adequate for the case of $04 \mathrm{~h}$. All statistical performance indices were improved at this case study, compared to Model A. This was due to the expansion of input variables vector by adding additional past electricity prices on the same hour. In fact, the assumption that electricity prices "contain" a periodicity effect was verified by this simulation.

TABLE III

PERFORMANCE INDICES

\begin{tabular}{|l|c|c|}
\hline \multirow{2}{*}{$\begin{array}{c}\text { Statistical index for AGFINN } \\
\text { (Model B) }\end{array}$} & \multicolumn{2}{c|}{ Testing Data sets } \\
\cline { 2 - 3 } & $\mathbf{2 2 h}$ & $\mathbf{0 4 h}$ \\
\hline Root mean square error (RMSE) & 7.3320 & 3.3089 \\
\hline Mean absolute percentage error (MAPE) (\%) & 4.5763 & 6.2654 \\
\hline Standard error of prediction (SEP) (\%) & 6.0593 & 7.4027 \\
\hline
\end{tabular}

Results shown at Table IV illustrate results from alternative methods. For this case study, an AFLS model was constructed with 25 rules for the case of $22 \mathrm{~h}$, while 20 rules were used for the case of $04 \mathrm{~h}$. The MLP NN retained the same network configuration, while under these conditions, ANFIS performed satisfactory, its performance however was achieved with a high computational cost, by utilizing two membership functions for each input variables and 64 fuzzy rules. ANFIS is a classic representative of TSK-based neurofuzzy systems. Its main drawback is that the number of fuzzy rules increases exponentially with respect to the number of inputs $n$.

TABLE IV

PERFORMANCE INDICES - COMPARISON

\begin{tabular}{|c|c|c|c|}
\hline Statistical index (22h) & AFLS & ANFIS & MLP \\
\hline Root mean square error (RMSE) & 7.9278 & 9.8380 & 11.6525 \\
\hline Mean absolute percentage error (MAPE) (\%) & 4.8062 & 6.1757 & 6.9578 \\
\hline Standard error of prediction (SEP) (\%) & 6.5517 & 8.1302 & 9.6298 \\
\hline Statistical index (04h) & AFLS & ANFIS & MLP \\
\hline Root mean square error (RMSE) & 4.6194 & 6.0034 & 8.7796 \\
\hline Mean absolute percentage error (MAPE) (\%) & 9.5050 & 12.3199 & 10.6214 \\
\hline Standard error of prediction (SEP) (\%) & 10.3345 & 13.4307 & 19.6418 \\
\hline
\end{tabular}

\section{Model C}

The objective of this model is to expand Model B, by adding the exogenous input of the forecasted electricity load. Thus, for electricity price modeling for a specific hour (i) and day (j), the following seven input variables have been considered:

\section{Target:}

Price $(i, j)$ : electricity price at the $i^{\text {th }}$ hour on the $(j)^{\text {th }}$ day,

\section{Inputs:}

Price $(i, j-1)$ : price at the $i^{\text {th }}$ hour on the $(j-1)^{\text {th }}$ day, Price $(i, j-2)$ : price at the $i^{\text {th }}$ hour on the $(j-2)^{\text {th }}$ day, Price $(i, j-3)$ : price at the $i^{\text {th }}$ hour on the $(j-3)^{\text {th }}$ day, Price $(i, j-7)$ : price at the $i^{\text {th }}$ hour on the $(j-7)^{\text {th }}$ day, Price $(i-1, j-1)$ : price at the $(i-1)^{\text {th }}$ hour on the $(j-1)^{\text {th }}$ day, Price $(i-2, j-1)$ : price at the $(i-2)^{\text {th }}$ hour on the $(j-1)^{\text {th }} d a y$, Load $(i, j)$ : electricity load at the $i^{\text {th }}$ hour on the $j^{\text {th }}$ day,

The complete results for the hours with minimum and maximum electricity price, for the AGFINN case are illustrated in Table V. The information related to weekly periodicity as well as the exogenous load parameter indeed resulted in an improved forecasting performance compared to previous case studies.

TABLE V PERFORMANCE INDICES

\begin{tabular}{|c|c|c|}
\hline \multirow{2}{*}{$\begin{array}{c}\text { Statistical index for AGFINN } \\
\text { (Model C) }\end{array}$} & \multicolumn{2}{c|}{ Testing Data sets } \\
\cline { 2 - 3 } & $\mathbf{2 2 h}$ & $\mathbf{0 4 h}$ \\
\hline Root mean square error (RMSE) & 6.8514 & 2.9988 \\
\hline Mean absolute percentage error (MAPE) (\%) & 4.2418 & 5.4832 \\
\hline Standard error of prediction (SEP) (\%) & 5.6621 & 6.7089 \\
\hline
\end{tabular}

Best results were produced by including 25 fuzzy rules for the case of $22 \mathrm{~h}$, while 20 rules were considered adequate for 
the case of $04 \mathrm{~h}$. Figures 3 and 4 illustrate the testing performances for minimum and maximum electricity price forecasting using Model C.

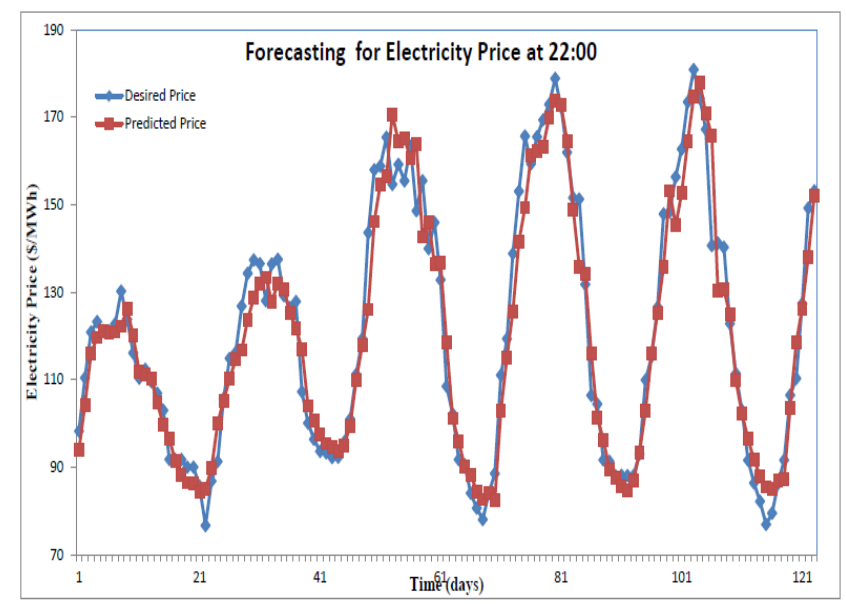

Fig. 3. Forecasting for Electricity Price at 22:00, (AGFINN-Model C)

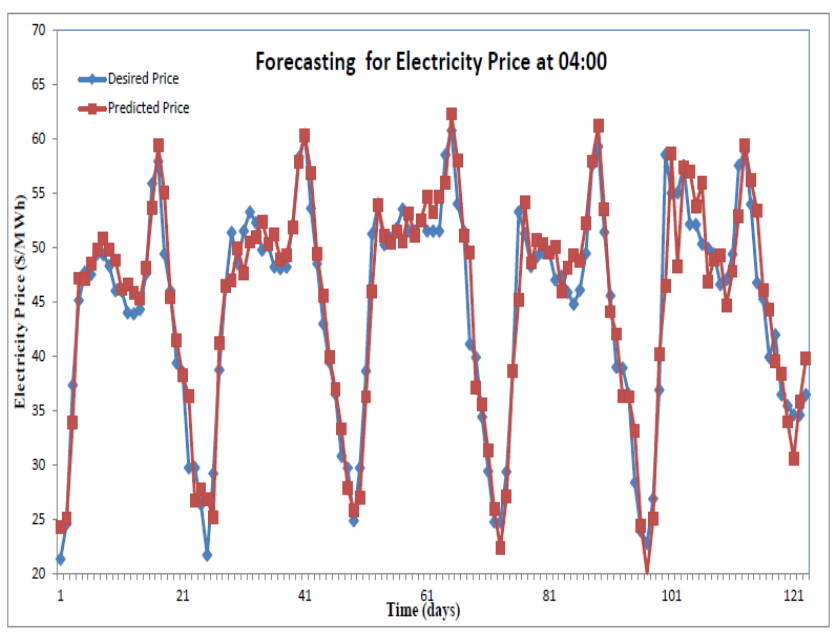

Fig. 4. Forecasting for Electricity Price at 04:00, (AGFINN-Model C)

Similarly, to previous case studies, AFLS, ANFIS and MLP NN have been applied to this specific case study and their performances are presented at Table VI. ANFIS's performance was achieved however with a huge computational cost, by utilizing 128 fuzzy rules.

TABLE VI

PERFORMANCE INDICES - COMPARISON

\begin{tabular}{|l|c|c|c|}
\hline Statistical index (22h) & AFLS & ANFIS & MLP \\
\hline Root mean square error (RMSE) & 7.7340 & 9.1584 & 11.4835 \\
\hline Mean absolute percentage error (MAPE) (\%) & 4.4775 & 5.3308 & 6.0115 \\
\hline Standard error of prediction (SEP) (\%) & 6.3915 & 7.5686 & 9.4901 \\
\hline \multicolumn{3}{|l|}{} \\
\hline Statistical index (04h) & AFLS & ANFIS & MLP \\
\hline Root mean square error (RMSE) & 4.3667 & 5.4409 & 8.0055 \\
\hline Mean absolute percentage error (MAPE) (\%) & 7.6772 & 8.3168 & 16.5878 \\
\hline Standard error of prediction (SEP) (\%) & 9.7692 & 12.1724 & 17.9098 \\
\hline
\end{tabular}

\section{CONCLUSIONS}

An approach is proposed in this paper for short-term electricity prices forecasting, based on an asymmetric neurofuzzy identification model. The application of the proposed approach to electricity prices forecasting on the ISO New England market is novel in terms of network architecture and forecasting performance. The effectiveness of this approach has been thoroughly assessed by comparing it with alternative neural or neurofuzzy techniques, via three case studies. Future research includes the incorporation in the modeling process additional exogenous parameters.

\section{REFERENCES}

[1] A.J. Conejo, J. Contreras, R. Espínola, M.A. Plazas, "Forecasting electricity prices for a day-ahead pool-based electric energy market", Int. J Forecasting, Vol. 21, No. 3, pp. 435-462, 2005.

[2] F. Ziel, R. Steinert, S. Husmann, "Efficient modeling and forecasting of electricity spot prices", Energy Econ, Vol. 47, pp. 98-111, 2015.

[3] C.J. Cuaresma, J. Hlouskova, S. Kossmeier, M. Obersteiner, "Forecasting electricity spot-prices using linear univariate time-series models", Applied Energy, Vol. 77, pp. 87-106, 2004.

[4] S. Anbazhagan, N. Kumarappan, "Day-ahead deregulated electricity market price classification using neural network input featured by DCT”, Int. J. Electr. Power Energy Syst., Vol. 37, pp. 103-109, 2012.

[5] R. Gareta, L.M. Romeo, A. Gil, "Forecasting of electricity prices with neural networks", Energy Conversion Management, Vol. 47, 1770$1778,2006$.

[6] Z. Yun, Z. Quan, S. Caixin, L. Shaolan, L. Yuming, S. Yang, "RBF neural network and ANFIS-based short-term load forecasting approach in real-time price environment", IEEE Trans Power Systems, Vo. 23, No. 3, pp. 853-858, 2008.

[7] A. Mohamed, M.E El-Hawary, "Mid-term electricity price forecasting using SVM", 2016 IEEE Canadian Conference on Electrical and Computer Engineering, 2016, Article number 7726765.

[8] Y.-Y. Hong, C.-Y. Hsiao, "Locational marginal price forecasting in deregulated electricity markets using artificial intelligence", IEE Proceedings: Generation, Transmission and Distribution, Vol. 149, No. 5, pp. 621-626, 2002.

[9] J.P. Catalao, H.M. Pousinho, V.M. Mendes, "Hybrid wavelet-PSOANFIS approach for short-term electricity prices forecasting", IEEE Trans Power Systems, Vol.26, No. 1, pp. 137-144, 2011.

[10] V.S. Kodogiannis, M. Amina, I. Petrounias, "A clustering-based fuzzy-wavelet neural network model for short-term load forecasting", Int. Journal of Neural Systems, Vol. 23, No. 5, 2013

[11] I. Rojas, H. Pomares, F.J. Fernandez, "A new methodology to obtain fuzzy systems autonomously from training data", IEEE conf. Fuzzy System, Vol. 1, pp. 527-532, 1999.

[12] V.S. Kodogiannis, T. Pachidis, E. Kontogianni, "An intelligent based decision support system for the detection of meat spoilage", Eng. Appl. of Artificial Intelligence, Vol. 34, pp. 23-36, 2014.

[13] V.S. Kodogiannis, A. Alshejari, "An adaptive neuro-fuzzy identification model for the detection of meat spoilage", Applied Soft Computing, Vol. 23, pp.483-497, 2014. 